This item was submitted to Loughborough's Research Repository by the author.

Items in Figshare are protected by copyright, with all rights reserved, unless otherwise indicated.

\title{
Maximisation of feasibility/stability regions of model predictive control for constrained linear systems
}

PLEASE CITE THE PUBLISHED VERSION

PUBLISHER

(C) Institution of Electrical Engineers

LICENCE

CC BY-NC-ND 4.0

REPOSITORY RECORD

Chen, Wen-Hua. 2008. "Maximisation of Feasibility/stability Regions of Model Predictive Control for Constrained Linear Systems". figshare. https://hdl.handle.net/2134/3851. 
This item was submitted to Loughborough's Institutional Repository (https://dspace.lboro.ac.uk/) by the author and is made available under the following Creative Commons Licence conditions.

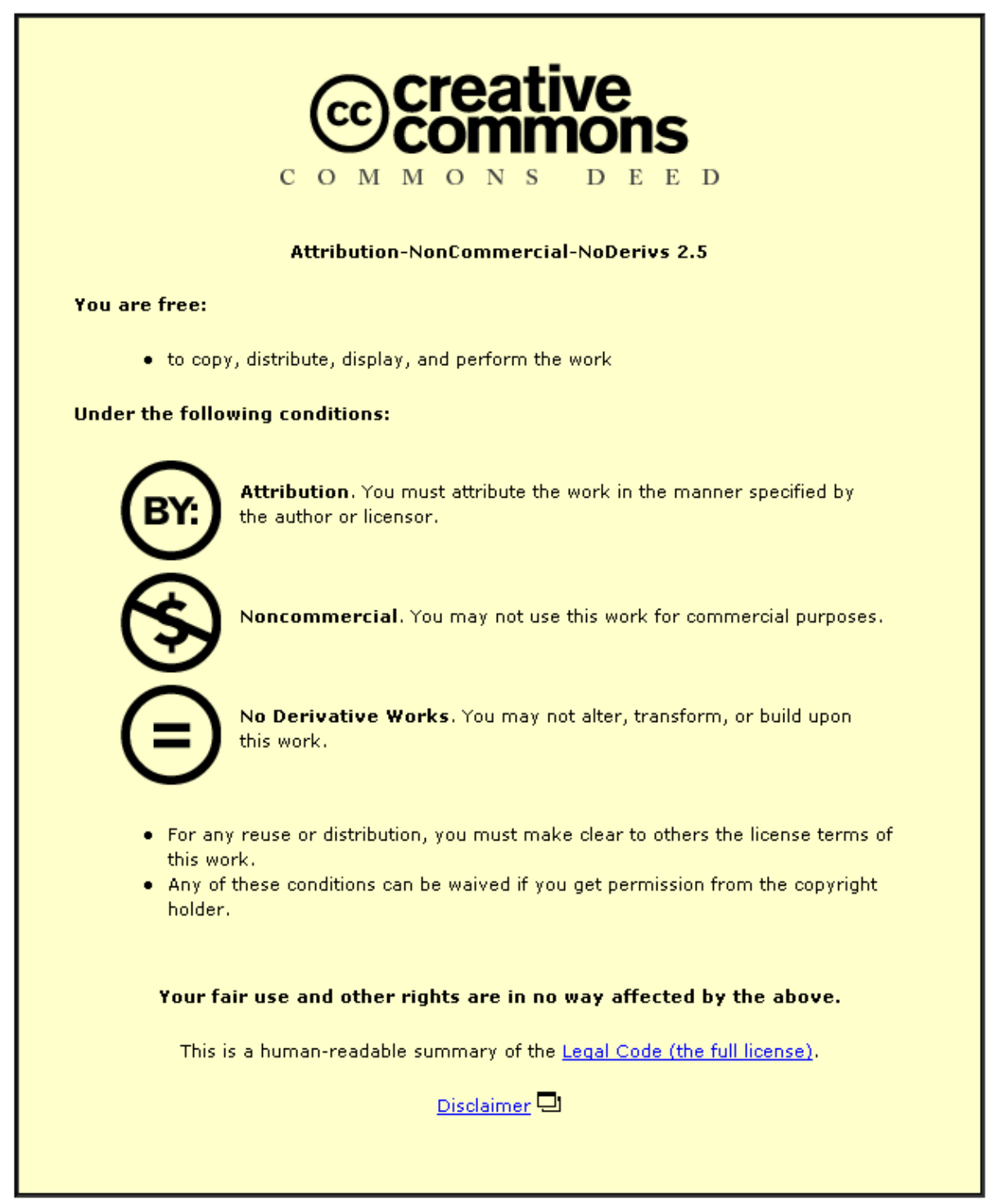

For the full text of this licence, please go to: http://creativecommons.org/licenses/by-nc-nd/2.5/ 


\title{
Maximisation of Feasibility/Stability Regions of Model Predictive Control for Constrained Linear Systems
}

\author{
Wen-Hua Chen \\ Department of Aeronautical and Automotive Engineering \\ Loughborough University \\ Loughborough, Leicestershire LE11 3TU, UK \\ email:w.chen@lboro.ac.uk
}

Key words: Model predictive control; stability; saturation; optimisation, LMI's

\begin{abstract}
Stability of model predictive control could be achieved by adding a terminal weighting term in a performance index and the feasibility/stability region largely depends on the choice of the terminal weighting term and associated terminal control laws. For constrained linear systems, different from existing methods where the stability region is estimated by its terminal region, a new method to estimate feasibility/stability regions directly is proposed using a new representation of the behaviour of MPC. A design procedure is then developed to determine the terminal term such that the feasibbility/stability region of MPC algorithms is as large as possible. Examples show that the stability region is greatly enlarged.
\end{abstract}




\section{Introduction}

Stability of model predictive control (MPC) has been investigated by many authors (see example, see recent review in [1]). To guarantee stability of MPC, the most widely used method is to add a terminal weighting term in the performance index and (or) impose constraints on terminal state. Recently these results are extended from linear systems to the nonlinear case; for example, see [1], [2].

For the engineering application of MPC, a stability condition on its own is not enough, and it's desired that the stability regions of the proposed MPC schemes are as large as possible. The choice of the terminal weighting term in the performance index affects the size of the stability region of MPC which may vary from the whole state space to nil. For an unconstrained linear system, it is shown that when the terminal weighting term in the performance index satisfies the so-called fake algebraic Riccati equation (FARE), MPC globally stabilises a linear system [3]. For a stable linear system with a convex input constraint, Rawing and Muske [4] points out that the closed-loop system is stable if the terminal weighting term covers the performance cost of the free system (no control action imposed after the terminal time). This control scheme is also equivalent to the case where an infinite horizon performance (state) cost and a finite horizon control cost are employed. The stability result for this kind of systems is easy to understand since the linear system is stable under zero control input which satisfies the input constraint. Recently, this kind of global stability result has been further extended to the so-called asymptotically null-controllable with bounded inputs (ANCBI) system which is stabilisable and has eigenvalues of modulus less than 1 or equal to one (for discrete-time systems) in [5].

For general constrained linear systems, it is unlikely to achieve global stability. A systematic method to determine the terminal weighting term to maximise the stability region of MPC has been developed by [6] and [7]. The underlying idea 
is to optimise the terminal linear control and the terminal weighting matrix in terms of the stability region. Most recently Lee and Kouvaritakis [8] proposes a new approach to relax the stability region based on eigenvector decomposition for a given linear stabilising gain. It is shown that it is possible to result in an infinite stability region in certain directions. For constrained nonlinear systems, optimisation based methods to determine the terminal weighting matrix have also been proposed in $[9,10]$.

The motivation to achieve a large stability region for an MPC algorithm is obvious since this implies that the MPC algorithm can work in a large range. However, although feasibility/stability regions are one of the main concerns of MPC, in almost all existing works (for example, see $[9,8,6,7,10]$ ) the terminal weighing term and associated terminal law are designed in terms of terminal regions rather than feasibility/stability regions themselves. The main difficulty in estimating the stability/feasibility region directly lies in that the hehaviour of MPC is very complicated since not only the constraints on input and state may be active but also an on-line optimisation solver is involved in loop. A method to estimate stability regions directly is presented in [11]. In the paper, different from conventional MPC schemes where a quadratic performance index is on-line optimised, a controller consisting of an LQ controller for unconstrained systems with infinite horizon and a sequence of perturbations on the LQ controller is proposed, and the on-linear optimiser tries to miminise the perturbations.

In view of the difficulty in direct estimation of stability regions for conventional MPC schemes, stability regions are approximately estimated by their terminal regions. It is relatively easy to estimate terminal regions since a terminal controller is, in general, a conventional controller. Although terminal regions are contained in feasibility/stability regions and thus can be used as a measure to estimate feasibility/stability regions, two factors affect the efficiency of the existing methods. One is the gap between terminal regions and feasibility/stability regions 
which results conservativeness in the estimation of stability/feasibility regions. As will be shown by the example in this paper, it could be very significant. The other is that the maximisation of terminal regions does not necessarily imply that feasibility/stability regions are maximised.

To avoid these problems, this paper reports a new approach to optimally choose the terminal weighting term and associated terminal control laws in terms of feasbility/stability regions of a MPC algorithm directly. This is achieved by using a new representation of the behaviour of MPC. The control sequence within the receding horizon yielded by an on-line optimisation solver on the basis of the state measurement is represented by a linear control law with different gains at each time instant in this paper. A new method to analyse the stability region of an MPC algorithm for linear constrained systems directly is proposed.This enables us to develop a procedure to determine the terminal weighting matrix to achieve the maximum feasibility/stability region. It is shown that this problem is well posed and can be formulated as a convex optimisation problem.

The remainder of this paper is organised as follows: Section 2 introduces the MPC scheme for constrained linear systems. The main results are reported in Section 3 where a method to estimate feasibility/stability regions directly and the procedure for optimisation of the terminal term in terms of feasibility/stability regions are developed. The proposed method is illustrated by a numerical example and compared with other existing methods in Section 4. Finally this paper is ended with conclusion in Section 5.

\section{MPC for constrained linear systems}

Consider a constrained discrete time system

$$
\left\{\begin{array}{l}
\boldsymbol{x}(k+1)=\boldsymbol{A} \boldsymbol{x}(k)+\boldsymbol{B} \boldsymbol{u}(k) \\
\boldsymbol{x}(0)=\boldsymbol{x}_{\mathbf{0}}
\end{array}\right.
$$


with control constraints

$$
\left|u_{i}\right| \leq \bar{u}_{i}, \quad i=1, \ldots m
$$

where $\boldsymbol{x} \in \mathbb{R}^{n}$ and $\boldsymbol{u}=\left[u_{1}, \cdots, u_{m}\right]^{T} \in \mathbb{R}^{m}$ are the state and control vectors respectively. For the sake of simplicity, only input constraints are considered in this paper but state constraints can be added using the techniques in [10] or [6].

MPC is, at state $\boldsymbol{x}(k)$ and time instant $k$, to find a control sequence $\boldsymbol{u}(k \mid k), \ldots$, $\boldsymbol{u}(k+N-1 \mid k)$ such that the quadratic performance index in moving horizon time frame

$$
\begin{aligned}
J(k)= & \boldsymbol{x}(k+N \mid k)^{T} \boldsymbol{P} \boldsymbol{x}(k+N \mid k)+ \\
& +\sum_{i=0}^{N-1}\left(\boldsymbol{x}(k+i \mid k)^{T} \boldsymbol{Q} \boldsymbol{x}(k+i \mid k)+\boldsymbol{u}(k+i \mid k)^{T} \boldsymbol{R u}(k+i \mid k)\right)
\end{aligned}
$$

is minimised and the control constraints (2) are satisfied where $N$ is the predictive length, $\boldsymbol{x}(k+i \mid k)$ denotes the prediction of the state at time instant $k+i$ based on the control $\boldsymbol{u}(k \mid k), \ldots, \boldsymbol{u}(k+i \mid k)$ and the state measurement $\boldsymbol{x}(k) . \boldsymbol{Q}>0$ (or $\boldsymbol{Q} \geq 0$ and $\left[\boldsymbol{A}, \boldsymbol{Q}^{1 / 2}\right]$ is detectable), $\boldsymbol{R}>0$ and $\boldsymbol{P}>0$ are the state, control and terminal weighting matrices respectively. The above MPC problem can be formulated as

$$
\min _{\boldsymbol{u}(k \mid k), \ldots, \boldsymbol{u}(k+N-1 \mid k)} J(k)
$$

subject to (1) and (2). The problem (4) is solved on-line using an optimisation solver, for example, quadratic programming (QP), and a control sequence $\boldsymbol{u}(k \mid k), \ldots, \boldsymbol{u}(k+N-1 \mid k)$ is yielded. Then the MPC law is determined by

$$
\boldsymbol{u}(k)=\boldsymbol{u}(k \mid k)) \text {. }
$$

Before developing our main results, some definitions are necessary.

Definition 1: Terminal region $\mathcal{V}$ is defined as a region where once the terminal state $\boldsymbol{x}(k+N \mid k)$ under the control $\boldsymbol{u}(k \mid k), \ldots, \boldsymbol{u}(k+N-1 \mid k)$ yielded by minimisation of the cost (3), arrives, there exists a control sequence $\boldsymbol{u}(k+N+i \mid k), i=$ $0,1, \ldots, \infty$, satisfying the constraints $(2)$, which steers the state to the origin. 
Definition 2: Feasibility region refers to a set of initial state points $x_{0}$ from which the optimal state trajectory $\boldsymbol{x}(i \mid 0), i=1, \ldots, N$ under the optimal openloop control sequence $\boldsymbol{u}(i \mid 0)), i=0,1, \cdots, N-1$ satisfying the constraints (2) yielded by solving the optimisation problem (4) arrives in the terminal region $\mathcal{V}$ at time instant $N$.

Remark 1: To ensure stability of the MPC, after the receding horizon, a terminal control law is used to stabilise the plant within a terminal region. However, this terminal control is never implemented and thus is a fictitious controller. It is only used for determining the terminal weighting term off-line and for stability analysis. In general, the feasibility region is larger than the terminal region.

There are two objectives in this paper: One objective is to estimate the stability/feasibility region of a MPC algorithm under a given performance index. The other objective is to develop a systematic method to determine the terminal weighting term in the performance index and provides guidance for MPC users to choose the performance index in terms of stability requirement.

The following previous results will be used in our development.

Lemma 1 [1, 2]: Consider the MPC scheme described in Section 2 for the constrained system (1) and (2) under the performance index (3). Suppose that there exists $\boldsymbol{u}(k)$ satisfying (2) such that

$$
\boldsymbol{x}(k+1)^{T} \boldsymbol{P} \boldsymbol{x}(k+1)-\boldsymbol{x}(k)^{T} \boldsymbol{P} \boldsymbol{x}(k)+\boldsymbol{x}(k)^{T} \boldsymbol{Q} \boldsymbol{x}(k)+\boldsymbol{u}(k)^{T} \boldsymbol{R u}(k) \leq 0
$$

holds for all $\boldsymbol{x}(k) \in \mathcal{V}$. Then the set $\mathcal{V}$ is a terminal region of the MPC algorithm. Moreover, if the MPC scheme is feasible at time instant 0 , it is feasible for all $k \geq 0$ and the closed-loop system under the MPC law stemming from (4) is asymptotically stable about the origin.

Remark 2: Lemma 1 indicates that every trajectory which starts from the feasibility region arrives at the origin under the proposed MPC scheme. According to the definition of the feasibility region and Lemma 1, the stability region under 
the MPC algorithm is equal to its feasibility region. Thus hereafter only stability region is considered.

Lemma 2 [6]: Suppose that the terminal weighting matrix $\boldsymbol{P}=\boldsymbol{W}^{-1}>0$ satisfies

$$
\left[\begin{array}{cccc}
-\boldsymbol{W} & \boldsymbol{W} \boldsymbol{A}^{T}+\boldsymbol{W}_{1}^{T} \boldsymbol{B}^{T} & \boldsymbol{W}^{T}\left(\boldsymbol{Q}^{1 / 2}\right)^{T} & \boldsymbol{W}_{1}^{T} \\
\boldsymbol{A} \boldsymbol{W}+\boldsymbol{B} \boldsymbol{W}_{1} & -\boldsymbol{W} & \mathbf{0} & \mathbf{0} \\
\boldsymbol{Q}^{1 / 2} \boldsymbol{W} & \mathbf{0} & -\boldsymbol{I} & \mathbf{0} \\
\boldsymbol{W}_{1} & \mathbf{0} & \mathbf{0} & -\boldsymbol{R}^{-1}
\end{array}\right] \leq 0
$$

and

$$
\left[\begin{array}{cc}
\boldsymbol{Y} & \boldsymbol{W}_{1} \\
\boldsymbol{W}_{1}^{T} & \boldsymbol{W}
\end{array}\right] \geq 0 \quad Y_{i i} \leq \bar{u}_{i}^{2}, \quad i=1, \ldots, m
$$

Then the optimisation problem (4) is always feasible for all $k \geq 0$ and for all initial state $\boldsymbol{x}_{0} \in \mathcal{V}$, defined by

$$
\mathcal{V}=\left\{\boldsymbol{x} \in \mathbb{R}^{n}: \boldsymbol{x}^{T} \boldsymbol{P} \boldsymbol{x} \leq 1\right\}
$$

Moreover, the MPC stemming from this optimisation problem exponentially stabilises the system for all initial state $\boldsymbol{x}_{0} \in \mathcal{V}$ while satisfying constraints (2)

Remark 3: It can be shown that the stability region estimated in Lemma 2, i.e., the set $\mathcal{V}$, is actually the terminal region. This is because conditions (7) and (8) imply that there exist a linear control law $\boldsymbol{u}(k)=\boldsymbol{K} \boldsymbol{x}(k)=\boldsymbol{W}_{1} \boldsymbol{W}^{-1} \boldsymbol{x}(k)$ satisfying the constraints (2) for all $\boldsymbol{x}(k) \in \mathcal{V}$ and a matrix $\boldsymbol{P}=\boldsymbol{W}^{-1}$ such that condition (6) is satisfied. It follows from Lemma 1 that the set $\mathcal{V}$ is a terminal set. Hence the terminal region is used in Lemma 2 as an estimation of the stability region. The similar method is also adopted in $[9,10]$. As will be shown in the example later, this estimation is very conservative. 


\section{Stability regions of MPC}

\subsection{Estimation of stability regions}

Let the control effort in each time instant be given by

$$
\boldsymbol{u}(k+i \mid k)=\boldsymbol{K}(k+i) \boldsymbol{x}(k) \quad \text { for } i=0, \ldots, N-1
$$

where $\boldsymbol{K}(k+i)$ is the control gain at time instant $k+i$. Putting the control efforts from $i=0$ to $N-1$ in a vector form yields

$$
\overline{\boldsymbol{U}}(k)=\overline{\boldsymbol{K}}(k) \boldsymbol{x}(k)
$$

where

$$
\overline{\boldsymbol{U}}(k)=\left[\begin{array}{c}
\boldsymbol{u}(k \mid k) \\
\vdots \\
\boldsymbol{u}(k+N-1 \mid k)
\end{array}\right], \quad \overline{\boldsymbol{K}}(k)=\left[\begin{array}{c}
\boldsymbol{K}(k) \\
\vdots \\
\boldsymbol{K}(k+N-1)
\end{array}\right]
$$

Thus the state at time instant $k+N$ driven by the control sequence $\boldsymbol{u}(k \mid k), \ldots$, $\boldsymbol{u}(k+N-1 \mid k)$ from the state $\boldsymbol{x}(k)$ is predicted by

$$
\begin{aligned}
\boldsymbol{x}(k+N \mid k)= & \boldsymbol{A}^{N} \boldsymbol{x}(k)+\boldsymbol{A}^{N-1} \boldsymbol{B} \boldsymbol{u}(k \mid k)+\ldots+\boldsymbol{A} \boldsymbol{B} \boldsymbol{u}(k+N-2 \mid k)+ \\
& +\boldsymbol{B} \boldsymbol{u}(k+N-1 \mid k) \\
= & \boldsymbol{A}^{N} \boldsymbol{x}(k)+\boldsymbol{\Gamma} \overline{\boldsymbol{U}}(k) \\
= & \boldsymbol{A}^{N} \boldsymbol{x}(k)+\boldsymbol{\Gamma} \overline{\boldsymbol{K}}(k) \boldsymbol{x}(k) \\
= & \left(\boldsymbol{A}^{N}+\boldsymbol{\Gamma} \overline{\boldsymbol{K}}(k)\right) \boldsymbol{x}(k)
\end{aligned}
$$

where

$$
\boldsymbol{\Gamma}=\left[\begin{array}{llll}
\boldsymbol{A}^{N-1} \boldsymbol{B} & \cdots & \boldsymbol{A} \boldsymbol{B} & \boldsymbol{B}
\end{array}\right]
$$

Theorem: Consider a discrete-time linear system (1) subject to the input constraints (2). Suppose that there exist $\boldsymbol{S}>0, \overline{\boldsymbol{S}}, \boldsymbol{W}>0$ and $\boldsymbol{W}_{1}$ such that 


$$
\begin{gathered}
{\left[\begin{array}{cc}
\boldsymbol{S} & \left(\boldsymbol{A}^{N} \boldsymbol{S}+\boldsymbol{\Gamma} \overline{\boldsymbol{S}}\right)^{T} \\
\boldsymbol{A}^{N} \boldsymbol{S}+\boldsymbol{\Gamma} \overline{\boldsymbol{S}} & \boldsymbol{W}
\end{array}\right] \geq 0,} \\
{\left[\begin{array}{cc}
\boldsymbol{Y} & \boldsymbol{S}_{i} \\
\boldsymbol{S}_{i}^{T} & \boldsymbol{S}
\end{array}\right] \geq 0, \quad Y_{j j} \leq \bar{u}_{j}^{2}, \quad i=0,1, \ldots, N-1}
\end{gathered}
$$

and $(7),(8)$ hold where

$$
\overline{\boldsymbol{S}}=\left[\begin{array}{c}
\boldsymbol{S}_{0} \\
\vdots \\
\boldsymbol{S}_{N-1}
\end{array}\right]
$$

Then when the terminal weighting matrix in the performance index (3) is determined by $\boldsymbol{P}=\boldsymbol{W}^{-1}$, the MPC optimisation problem (4) subject to (1),(2) and $\boldsymbol{x}(k+N \mid k) \in \mathcal{V}$ is feasible for all $k \geq 0$ and all initial state $\boldsymbol{x}_{\mathbf{0}}$ within the set

$$
\mathcal{M}=\left\{\boldsymbol{x} \in \mathbb{R}^{n}: \boldsymbol{x}^{T} \boldsymbol{Z} \boldsymbol{x} \leq 1\right\},
$$

where $\boldsymbol{Z}=\boldsymbol{S}^{-1}$ and the closed-loop system is asymptotically stable about the origin with the stability region $\mathcal{M}$.

Proof: The terminal state $\boldsymbol{x}(N \mid 0)$ arrives in the set $\mathcal{V}$ in $(9)$ if

$$
\boldsymbol{x}(N \mid 0)^{T} \boldsymbol{P} \boldsymbol{x}(N \mid 0) \leq 1
$$

where $\boldsymbol{P}$ is the terminal weighting matrix. Substituting (13) into (19) obtains

$$
\boldsymbol{x}_{0}^{T}\left(\boldsymbol{A}^{N}+\boldsymbol{\Gamma} \overline{\boldsymbol{K}}(0)\right)^{T} \boldsymbol{P}\left(\boldsymbol{A}^{N}+\boldsymbol{\Gamma} \overline{\boldsymbol{K}}(0)\right) \boldsymbol{x}_{0} \leq 1
$$

Condition (20) is satisfied for all $\boldsymbol{x}_{\mathbf{0}} \in \mathcal{M}$ in (18) if there exists a matrix $\boldsymbol{Z}$ such that

$$
\left(\boldsymbol{A}^{N}+\boldsymbol{\Gamma} \overline{\boldsymbol{K}}(0)\right)^{T} \boldsymbol{P}\left(\boldsymbol{A}^{N}+\boldsymbol{\Gamma} \overline{\boldsymbol{K}}(0)\right) \leq \boldsymbol{Z},
$$

which is equivalent to that

$$
\left[\begin{array}{cc}
\boldsymbol{Z} & \left(\boldsymbol{A}^{N}+\boldsymbol{\Gamma} \overline{\boldsymbol{K}}(0)\right)^{T} \\
\boldsymbol{A}^{N}+\boldsymbol{\Gamma} \overline{\boldsymbol{K}}(0) & \boldsymbol{P}^{-1}
\end{array}\right] \geq 0 .
$$


Using the transform

$$
\begin{array}{r}
\boldsymbol{W}=\boldsymbol{P}^{-1}>0 ; \\
\boldsymbol{S}=\boldsymbol{Z}^{-1}>0 ; \\
\overline{\boldsymbol{S}}=\overline{\boldsymbol{K}}(0) \boldsymbol{S}=\overline{\boldsymbol{K}}(0) \boldsymbol{Z}^{-1},
\end{array}
$$

condition (22) becomes (15).

The control $\boldsymbol{u}(i \mid 0), i=0, \ldots, N-1$ satisfies the input constraints (2) if

$$
\left|\boldsymbol{K}_{j}(i) \boldsymbol{x}_{\mathbf{0}}\right| \leq \bar{u}_{j}, \quad \text { for } j=1, \ldots, m, i=0,1, \ldots, N-1
$$

hold where $\boldsymbol{K}_{j}(i)$ denotes the $j$ th row of the control gain $\boldsymbol{K}$ at time instant $i$.

For all $\boldsymbol{x}_{\mathbf{0}} \in \mathcal{M}$ in (18), condition (26) is satisfied if the following matrix inequalities hold

$$
\left[\begin{array}{cc}
\boldsymbol{Y} & \boldsymbol{K}(i) \\
\boldsymbol{K}(i) & \boldsymbol{Z}
\end{array}\right] \geq 0, \quad Y_{j j}=\bar{u}_{j}^{2}, \quad i=0,1, \ldots, N-1
$$

Using the transform in (23), (27) is implied by (16). For a constrained linear system (1), Lemma 2 and Remark 3 state that the set $\mathcal{V}$ is a terminal set if conditions (7) and (8) are satisfied.

Therefore the conditions (15) and (16) guarantee that for every state trajectory starts from an initial state $\boldsymbol{x}_{\mathbf{0}}$ within the set $\mathcal{M}$ there exits a control sequence render the state arrives in the terminal region $\mathcal{V}$ at time instant $N$. Conditions (7) and (8) ensure that there exists a terminal control satisfying the control constraints which can steer every state within the terminal set $\mathcal{V}$ to the origin. Hence the result follows from Lemma 1 and 2. QED

Remark 5: It should be noticed that the control sequence in MPC is yielded by online solving a constrained optimisation problem. Certainly, MPC under input constraints is highly nonlinear. However all the possible control sequences in MPC can be represented by a linear controller with different gains at each time instant 
as in (11). This is because for any sequence $\boldsymbol{u}(k \mid k)^{*}, \ldots, \boldsymbol{u}(k+N-1 \mid k)^{*}$ yielded by the on-line optimisation solver, there exist a set of control gains $\boldsymbol{K}(k)^{*}, \ldots, \boldsymbol{K}(k+$ $N-1)^{*}$, with the state measurement $\boldsymbol{x}(k)$, such that

$$
\begin{aligned}
\boldsymbol{K}(k)^{*} \boldsymbol{x}(k) & =\boldsymbol{u}(k \mid k)^{*} \\
\vdots & \\
\boldsymbol{K}(k+N-1)^{*} \boldsymbol{x}(k) & =\boldsymbol{u}(k+N-1 \mid k)^{*}
\end{aligned}
$$

Thus a tight estimation of the stability region for MPC should be given by the method developed in this paper.

\subsection{Choice of the terminal weighting}

The problem for maximisation of the stability region in terms of the terminal weighing term can be formulated as a convex optimisation problem

$$
\min _{\boldsymbol{S}, \overline{\boldsymbol{S}}, \boldsymbol{W}, \boldsymbol{W}_{1}} \log \left(\operatorname{det}\left(\boldsymbol{S}^{-1}\right)\right) \text { subject to }(15),(16),(7),(8) \boldsymbol{S}>0, \boldsymbol{W}>0
$$

Remark 4: The technique developed in [10] can be used to further enlarge the stability region. That is, by choosing the stability region as

$$
\mathcal{M}=\left\{\boldsymbol{x} \in \mathbb{R}^{n}: \boldsymbol{x}^{T} \boldsymbol{Z} \boldsymbol{x} \leq \mu\right\},
$$

a new tuning knob $\mu$ is introduced. As shown in [10] the stability region may be significantly enlarged. This tuning knob also can be chosen according to the trade off between the achieved performance and the size of the stability region (see [10] for detail). After the optimal terminal weighting matrix and the associated terminal control law is found by solving the above optimisation problem, a more accurate stability region estimation can be obtained by using eigenvalue techniques developed in [8]. 


\section{An Illustrative Example}

Consider a second order system

$$
\left\{\begin{array}{l}
\dot{x}_{1}=0.875 x_{1}+1.125 x_{2}+u \\
\dot{x}_{2}=0.375 x_{1}+1.625 x_{2}
\end{array}\right.
$$

with control constraint

$$
|u| \leq 1
$$

This is an unstable plant with one pole outside the unit circle. The weighting matrices in the performance index (3) are chosen as

$$
\boldsymbol{Q}=\left[\begin{array}{cc}
10 & 0 \\
0 & 10
\end{array}\right] ; \quad R=1
$$

We now want to choose the terminal weighting matrix, $\boldsymbol{P}$, in the performance index (3) such that the stability region is as large as possible and also estimate the final stability region of the resultant MPC.

When the predictive length is chosen as 3 , ie., $\mathrm{N}=3$, solving the optimisation problem in (28) yields the feasibility/stability region

$$
\mathcal{M}=\left\{\boldsymbol{x} \in \mathbb{R}^{n}: \boldsymbol{x}^{T}\left[\begin{array}{cc}
2.5703 & 8.0266 \\
8.0266 & 25.1559
\end{array}\right] \boldsymbol{x} \leq 1\right\} .
$$

which is shown by the solid ellipsoid in Figure 1.

The stability region of the MPC scheme increases when a longer predictive length, $N$, is used. For example, if the predictive length is chosen as 4 , the stability region is shown by the dashed ellipsoid in Figure 1, which is larger than that when $N=3$.

For comparison, the method developed in [6] is also tried on this example. Following the procedure, the maximum stability region yielded by this method is given by

$$
\mathcal{V}=\left\{\boldsymbol{x} \in \mathbb{R}^{n}: \boldsymbol{x}^{T}\left[\begin{array}{cc}
32.4635 & 91.9909 \\
91.9909 & 392.8153
\end{array}\right] \boldsymbol{x} \leq 1\right\}
$$




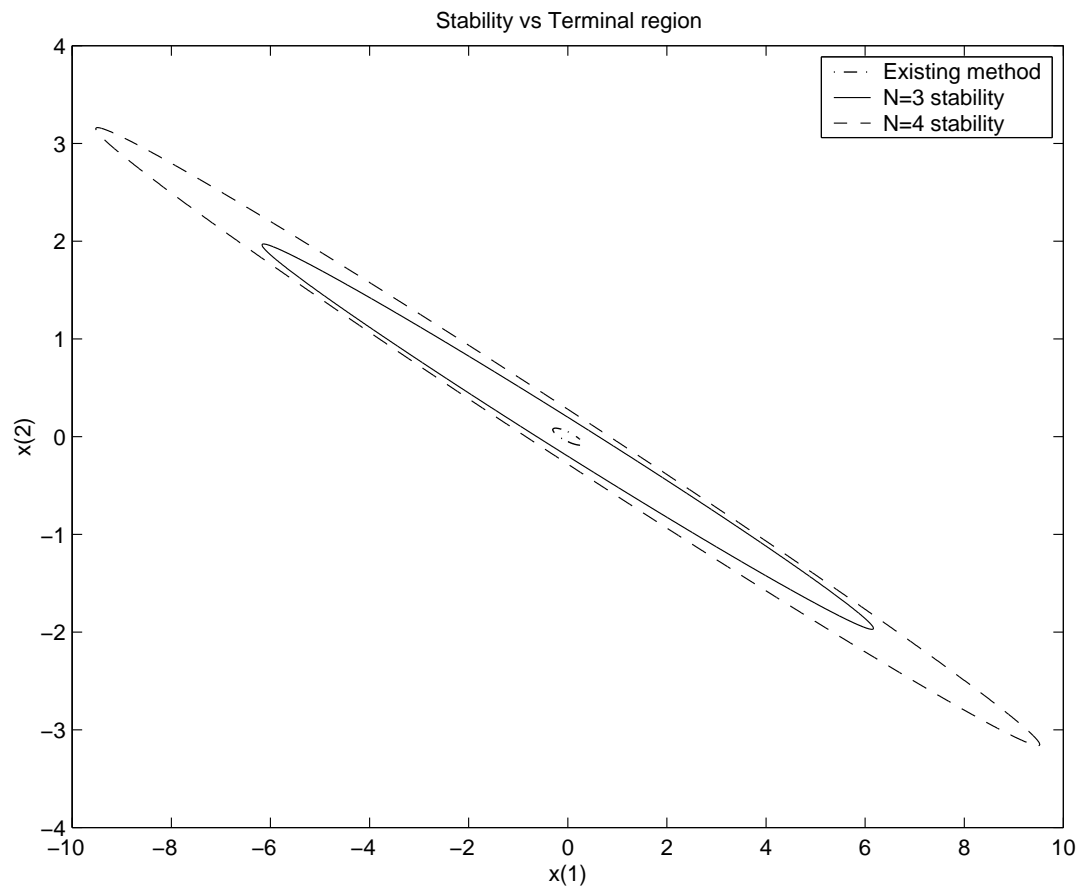

Figure 1: Stability regions and terminal region

which is shown by the dashed-dot ellipsoid in Figure 1. The stability region given by this method is only a very small part of the stability region $\mathcal{M}$ yielded by our method.

\section{Conclusion}

This paper presents a new method to estimate the feasibility/stability region which gives much less conservative than current methods. Based on this, a new method to determine the terminal weighting term in the performance index of MPC offline such that the stability/feasibility region is as large as possible is developed. It overcomes deficiencies of currently existing methods where the terminal weighing term is chosen based on the terminal region rather than the stability region. The illustrative example shows the efficiency of the proposed off-line design pro- 
cedure for MPC which provides guidance for engineers wishing to apply MPC for constrained linear systems.

\section{References}

[1] D.Q. Mayne, J.B. Rawlings, C.V. Rao, and P.O.M. Scokaert. Constrained model predictive control: stability and optimality. Automatica, 36:789-814, 2000.

[2] Wen-Hua Chen, D. J. Ballance, and J. O'Reilly. Model predictive control of nonlinear systems: computational burden and stability. IEE Proceedings Part D: Control Theory and Applications, 147(4):387-394, 2000.

[3] R. R. Bitmead, M. Gevers, and V. Wertz. Adaptive Optimal Control: The Thinking Man's GPC. Prentice-Hall, New York, 1990.

[4] J. B. Rawlings and K. R. Muske. The stability of constrained receding horizon control. IEEE Trans. on Automatic Control, 38(10):1512-1516, 1993.

[5] M. Gianneli A. Casavola and E. Mosca. Global predictive regulation of nullcontrollable input-saturated linear systems. IEEE Transactions on Automatic Control, 44(11):2226-2230, 1999.

[6] J.-W. Lee, W. H. Kwon, and J. Choi. On stability of constrained receding horizon control with finite terminal weighting matrix. Automatica, 34(12):1607$1612,1998$.

[7] J.-W. Lee. Exponential stability of constrained receding horizon control with terminal ellipsoid constraints. IEEE Transactions on Automatic Control, $45: 83-88,2000$. 
[8] Y.I. Lee and B. Kouvaritakis. Stabilizable regions of receding horizon predictive control with input constraints. Systems \& Control Letters, 38:13-20, 1999.

[9] H. Chen and F. Allgöwer. A quasi-infinite horizon nonlinear model predictive control scheme with guaranteed stability. Automatica, 34(10):1205-1217, 1998.

[10] Wen-Hua Chen, D. J. Ballance, and J. O'Reilly. Optimisation of terminal domains of nonlionear MPC via LMI methods. In Proceedings of American Control Conference, pages 2067-2072, Arlington, USA, 2001.

[11] M. Cannon B. Kouvaritakis and J.A. Rossiter. Who needs QP for linear MPC anyway? Automatica, 2001. To appear. 